UCRL - JC- 104448

PREPRINT

\title{
Success with an Automated Computer Control System
}

\section{L. Roberts and T. L. Moore}

Center for Accelerator Mass Spectrometry Lawrence Livernore National Laboratory

Livermore, California USA

This paper was prepared for submittal to

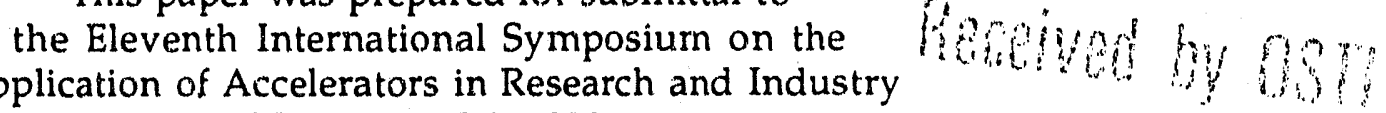
November 5-9, 1990

Denton, Texas

September 1990
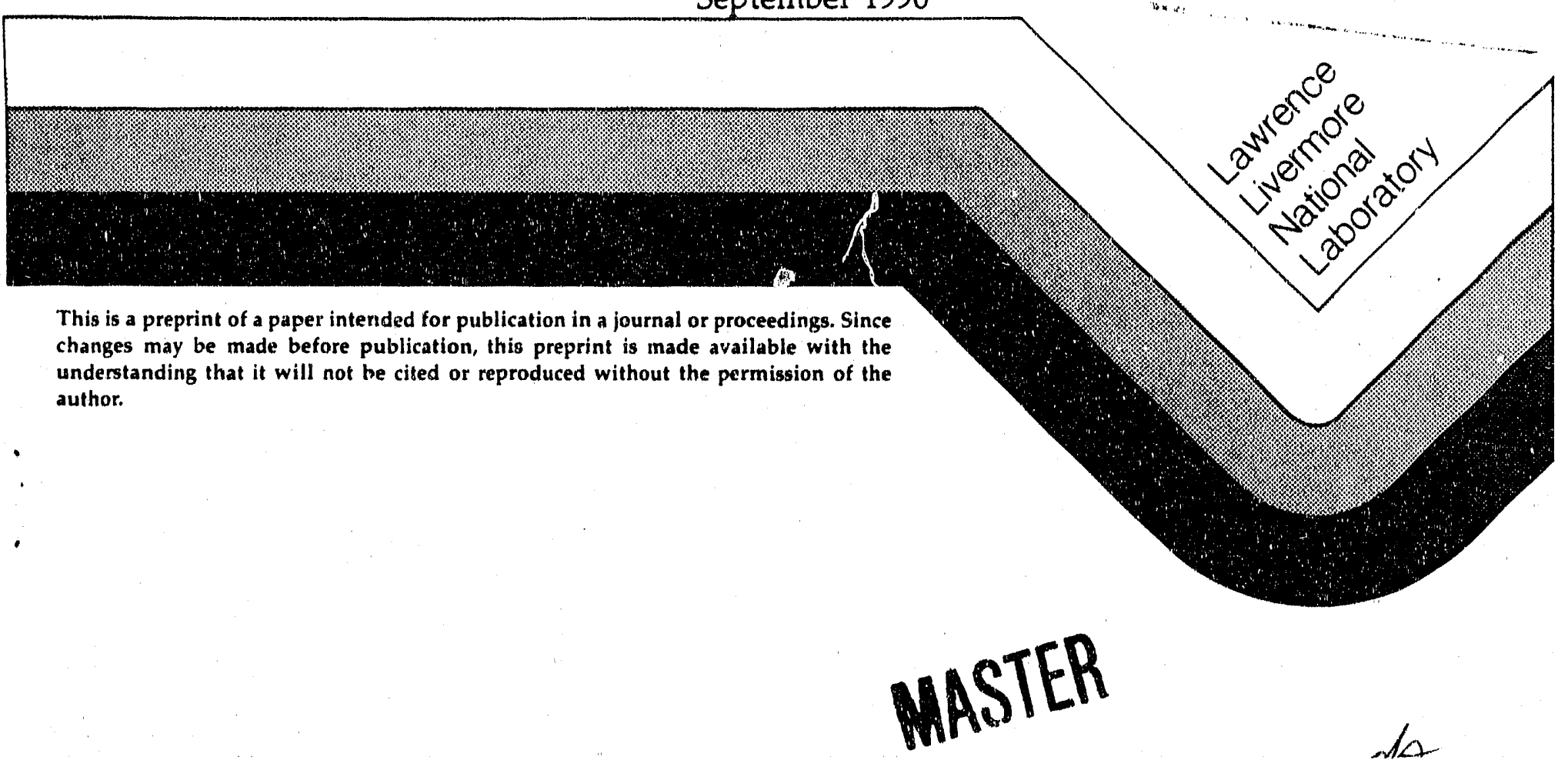

OHETRIBUTION DF TIIS ROCUMEXT IS UNLIMITED 
DISCI.AIMER

This document was prepared as an account of work sponsored by an agency of the Inited Situtes Government. Neither the Uaited State.j Government nor the University of California nor any of their employees, makes uny warranty, express or implied, or assumes uny lexul liability or rewponsibility for the accuracy, completeness, or usefulness of any information, apparatus, product, or process disclosed, or represents that its use nould not infringe privately owned rights. Reference herein to any spesific commercial products, precess, or service by trade name, trademark, munufacturer, or otherw ise, does not necessarily constitute or imply its endorsement, recommendation. or favoring by the United States Government or the Iniversity of California. The views and opinions of authors expressed herein do not necessurily state or reflect those of the I!nited States Corernment or the University of Calliornia, and shall not he used for advertising or product endoriement purposes.

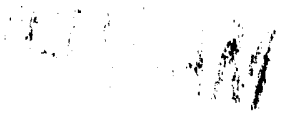


UCRL-JC--104448

DE91 001759

\title{
Success with an Automated Computer Control System
}

\author{
M.L. Roberts and T.L. Moore \\ Center for Accelerator Mass Spectrometry \\ Lawrence Livermore National Laboratory \\ Livermore, CA 94550 USA
}

September 1, 1990

\begin{abstract}
LLNL has successfully implemented a distributed computer control system for automated operation of an FN tandem accelerator. The control system software utilized is the Thaumaturgic Automated Control Logic (TACL) written by the Continuous Electron Beam Accelerator Facility and co-developed with LLNL. Using TACL, accelerator components are controlled through CAMAC using a two-tiered structure. Analog control and measurement are at 12 or 16 bit precision as appropriate. Automated operation has been implemented for several nuclear analytical techniques including hydrogen depth profiling and accelerator mass spectrometry. An additional advantage of TACL lies in its expansion capabilities. Without disturbing existing control definitions and algorithms, additional control algorithms and display functions can be implemented quickly.
\end{abstract}




\section{Introduction}

For the past three years, the physics department of the Lawrence Livermore National Laboratory has operated a tandem Van de Graaff laboratory. The laboratory was built primarily for multidisciplinary research with ion beams. Original design goals for the facility included low operating and staffing costs, ability to shift rapidly from one experiment to the next, and precise operation and control of the accelerator and related components. To meet these design goals, a distributed computer system operating through CAMAC interface was selected and has been implemented. This control system has demonstrated cost and scheduling advantages in construction and upgrades, has allowed activation of geographically dispersed equipment, and has permitted rapid switching from one experiment to the next. In this paper, the control system will be described with regard to the underlying design philosophy, system structure, control software, and beamline examples with specific emphasis on several new features implemented since previous descriptions of the control system[1].

\section{Control System}

\subsection{Underlying Design Philosophy}

The intended mode of operation for the tandem laboratory was for a scientist/researcher to operate the accelerator in a computer-assisted fashion, i.e., all tuning and setting of the accelerator would be done through the control system with the system maintaining the machine precisely in the configuration selected. All control of items involving safety or requiring high speed (such as radiation interlocks, vacuum interlocks, high voltage interlocks, fast terminal stabilization, etc.) are implemented outside the computer control system. Radiation protection is provided by a separate computer monitored radiation safety system. Vacuum and high voltage interlocks are hardwired. Terminal stabilization is accomplished using a fast analog feedback system. The control system merely monitors the state of the accelerator, while having the ability to change that state in response to a request from an operator or data acquisition system. Also, information obtained from high frequency devices (e.g. beam profile monitors and Faraday 
cups) is kept outside of the control system with control of such diagnostics through the control system.

\subsection{System Structure}

The accelerator and related components are controlled through a two-tiered hierarchical system of supervisory and local computers distributed throughout the laboratory and interconnected using an Ethernet Local Area Network (LAN). A schematic of the system is shown in Figure 1. In the present hierarchy, the supervisory computer serves as the programming resource for the entire control systen, coordination, and is the primary operator interface, while the local computers perform I/O and formatting of CAMAC data, bi-directional pass-through of parameters to the supervisor, and execute slosed-loop control of various local systems.

The current supervisory computer is a Hewlett-Packard 9000 series model 834 computer with a high resolution large (1280 $\times 1024$ pixels, 19 in) color monitor. We have recently added an additional supervisory computer. This second supervisory computer is a Hewlett-Packard 9000 series model 340 computer mounted on a roll-around cart and connected to the main supervisory computer via a LAN line. This extra supervisory computer is in essence a portable control console, that can be located anywhere in the lab. Remote testing and control of the accelerator and related components from the data acquisition and/or construction areas will be possible.

Currently there are three local computers geographically distributed throughout the laboratory. Use of multiple local computers allowed construction and testing of subsystems prior to integration into the complete system, placed computers near devices being controlled, and permitted truly parallel processing in the execution of control algorithms. Placing computers near devices being controlled minimizes extensive cableways and long cable runs, improving the overall analog signal to noise ratio. The first local computer, a Hewlett-Packard 9000 series model 319 computer, is used to control most of the ion sources and associated power supplies. The second local computer, also a Hewlett-Packard 9000 series model 319 computer, is used to control the low energy beam transport elements as well as some of the 
ion source power supplies. The third local computer is located at the high energy end of the tandem accelerator and is used to control the HV generator as well as the high energy beam transport elements. Because of the large number of components and control loops at the high energy end of the accelerator, this third local computer is a Hewlett-Packard 9000 series model 340 computer. A fourth local computer will be added shortly to contrcl the second switching magnet and related components.

The local computers interface to the controlled equipment via the General Purpose Interface Bus to CAMAC standard instrumentation. Commercially available digital to analog converters, analog to digital converters, and digital input and output registers comprise the bulk of the inst:umentation used. Analog measurements are made at either 12 or 16 bit precision as appropriate. At present, there are approximately 100 analog output channels, 125 analog input channels, and 1.50 digital input and output channels in the system.

Notably absent from the above discussion is any identified data-acquisition computer. Each beamline or experiment provides its own data acquisition equipment. Any interaction of the a data-acquisition system with the control system is through the LAN, CAMAC, or not at all. An example of an experiment in which interaction between the control system and a data-acquisition system occurs will ve given later (see Section 3.1).

\subsection{Control Software}

The control systern utilizes the Thaumaturgic Automated Control Logic (TACL) software package. TACL was written at the Continuous Electron Beam Accelerator Facility (CEBAF) and co-developed with LLNL[2]. TACL is written entirely in the $C$ language and operates under UNIX. Continuous development and expansion of the code is being preformed by Bork $[3]$ and co-workers at CEBAF.

TACL contains two editors and several run-time programs. Both of the editors are menu/icon driven. The first editor is called the system or logic editor and is further divided into two functional sub-parts. The first sub-part of the logic editor is used to define all hardware interfaces in the system 
including all LAN connections between computers, CAMAC crate addresses, module loading, and signal name definitions.

The second sub-part of the logic editor defines the data flow paths and algorithms used for system control. In the same manner as one would draw a block or schematic diagram, control algorithms and data flows are described by the user to the control system in graphical form via function blocks and ties. In the effort it takes to describe the control process, the user has done the programming without writing a single line of computer code. If a special function is desired, however, a simple module of $C$ code can be written and added to the function list. After the user has defined the control algorithms, TACL takes that information and stores it in a system file which is unique to the defined control system.

The second editor is called the display editor and is used to develop the customized display screens for operator monitoring and control of the system. The display editor is similar to the logic editor in that the user manipulates symbols and parameter fields to develop useful display configurations. Elements of the display include picture and/or schematic type drawings, toggle symbols, push-buttons, numeric data boxes, lines, and labels. The primary limit to these displays is the imagination of the user. We have developed our own custom display screens oriented to the specific functions we wish to perform. Figure 4 shows the display editor being used to create a customized display picture for the accelerator mass spectrometry beam line.

At run-time, TACL takes the files generated by the logic editor and derives the necessary database and relations to control the accelerator and related components. Display parameters are automatically li.ıked to the operating algorithms through variable name references and displayed on the operators screen. Operator interface is through a mouse, keyboard, or nine-knob knob box. Using the mouse, one can toggle certain devices (such as Faraday cups) or select an analog parameter to be entered through the keyboard. The nine-knob knob box can have its knobs assigned and reassigned to any analog parameter in the system, providing for continuous adjustment of those parameters. Once a specific accelerator state is established by tuning, parameters can be saved and recalled at a later time. 


\section{Beamline Examples}

\subsection{Hydrogen Profiling}

A hydrogen profiling beamline has been installed in the tandem accelerator and is a good example of the interaction of a data-acquisition system with the control system. A detailed description of the experiment can be found elsewhere[4], but the beamline exists primarily to measure the hydrogen depth profile in silicate candidate materials for nuclear waste cladding. The accelerator provides a ${ }^{19} \mathrm{~F}$ beam for depth profiling via the ${ }^{19} \mathrm{~F}(\mathrm{p}, \alpha \gamma){ }^{16} \mathrm{O}$ resonance technique. Control of the beamline is by a stand-alone data acquisition computer that executes an automatic excitation function sequence to produce a depth profile. After each energy point, the data acquisition computer requests a new energy from the tandem accelerator by inserting a word into memory of a CAMAC module. The tandem control system reads the CAMAC module and. upon recognizing that a new energy has been requested, automatically scales the terminal voltage and high energy transport elements as required. After the appropriate operating state has been achieved, the control system returns a logic signal to the data acquisition computer via an output register; the data acquisition system then accumulates data until a new energy is required.

\subsection{Accelerator Mass Spectrometry}

Accelerator mass spectrometry (AMS) is a high sensitivity technique for the detection of numerous long-lived radionuclides at extremely low concentrations. In the AMS measurement mode, the tandem functions as a mass spectrometer that is sufficiently stable and precise to measure isotope ratios of various unknowns to one part in 1014-16. The present use of this measurement tool at LLNL is primarily in archeology, the geosciences, and biomedical research[5]. To aid in initial tune-up of both the rare and abundant isotopes during AMS measurement, we have implemented into the control system a so called "flat-topping" routine. This routine is integrated into TACL in the form of a "user process" by which the control software allows execution of code which is not a standard part of the control system. In our case, "flat-topping" involves slewing a selected optical 
element over some suitable range and plotting the value of that element against either current from some arbitrary Faraday cup or counts from a detector. "Flat-topping" allows an the experimenter to precisely set the optimum value of selected elements.

\subsection{Others}

Other research preformed to date at the facility has included experiments in basic nuclear physics, nuclear chemistry, and nucleosynthesis. While none of data acquisition systems of these experiments have had a direct interaction with the computer control system, all experiments have benefited by the ease in operation provided by the control system including the capability to quickly switch from one ion species to the next and to make quick changes in incident beam energy.

\section{Conclusion}

We have successfully implemented a computer control system on a tandem Van de Graaff accelerator. We have found the present control system to be quick to install, logical to implement, and easy to modify. We have even begun to rethink traditional accelerator operating procedures to include unattended operation. While specific problems have occurred operating a distributed system of low level electronics in the high noise environment of a megavolt system that occasionally arcs over, most problems have been largely hardware related or problems of implementation and not the fault of the computer control system. We believe this control system is a viable cost effective way to implement controls in a large range of applications. 


\section{References}

[1] M.L. Roberts, T.L. Moore, R.S. Hornady, and J.C. Davis, "Distributed Computer Control at the LLNL Tandem", Submitted to Nuclear Instr'ments and Methods, February (1990).

[2] T.L. Moore, "Distributed Computer Controls for Accelerator Systems", Nuclear Instruments and Methods, B40/41 (1989) 984-987.

[3] R.G. Bork, "The CEBAF control system Architecture", Proceedings of the 1987 IEEE Particle Accelerator Conferense: Accelerator Engineering and Technology (1987) 523-525.

[4] R.S. Hornady, Proceedings of this conference.

[5] J.C. Davis, I.D. Proctor, J.R. Southon, M.W. Caffee, D.W. Heikkinen, M.L. Roberts, T.L. Moore, K.W. Turteltaub, D.E. Nelson, D.H. Loyd, J.S. Vogel, "LLNL/UC AMS Facility and Research Program", Submitted to Nuclear Instruments and Methods, April (1990).

\section{Figure Captions}

Figure 1. Block diagram of the distributed control system.

Work performed under the auspices of the U.S. Department of Energy at the Lawrence Livermore National Laboratory under contract W-7405-Eng-48. 


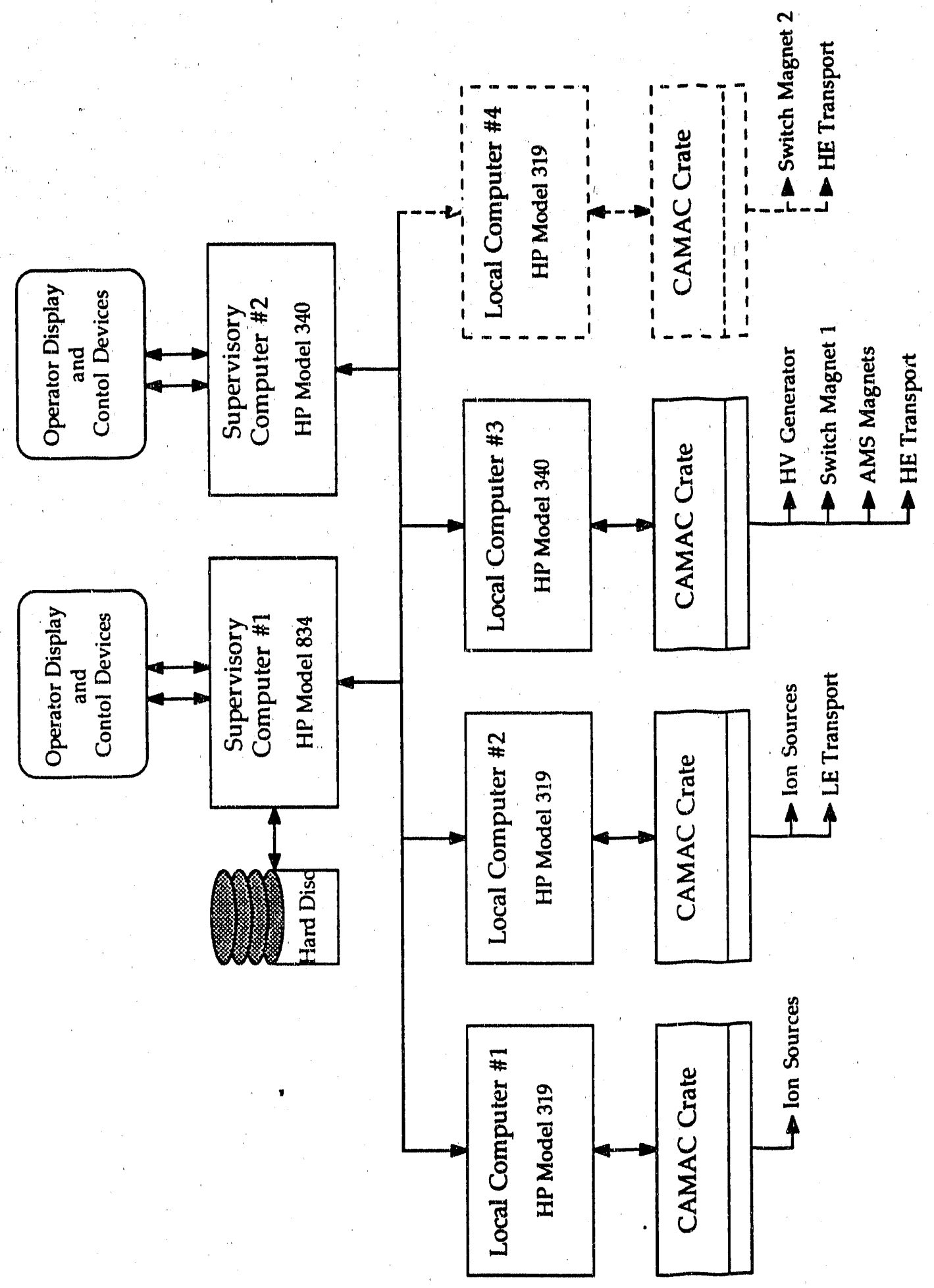



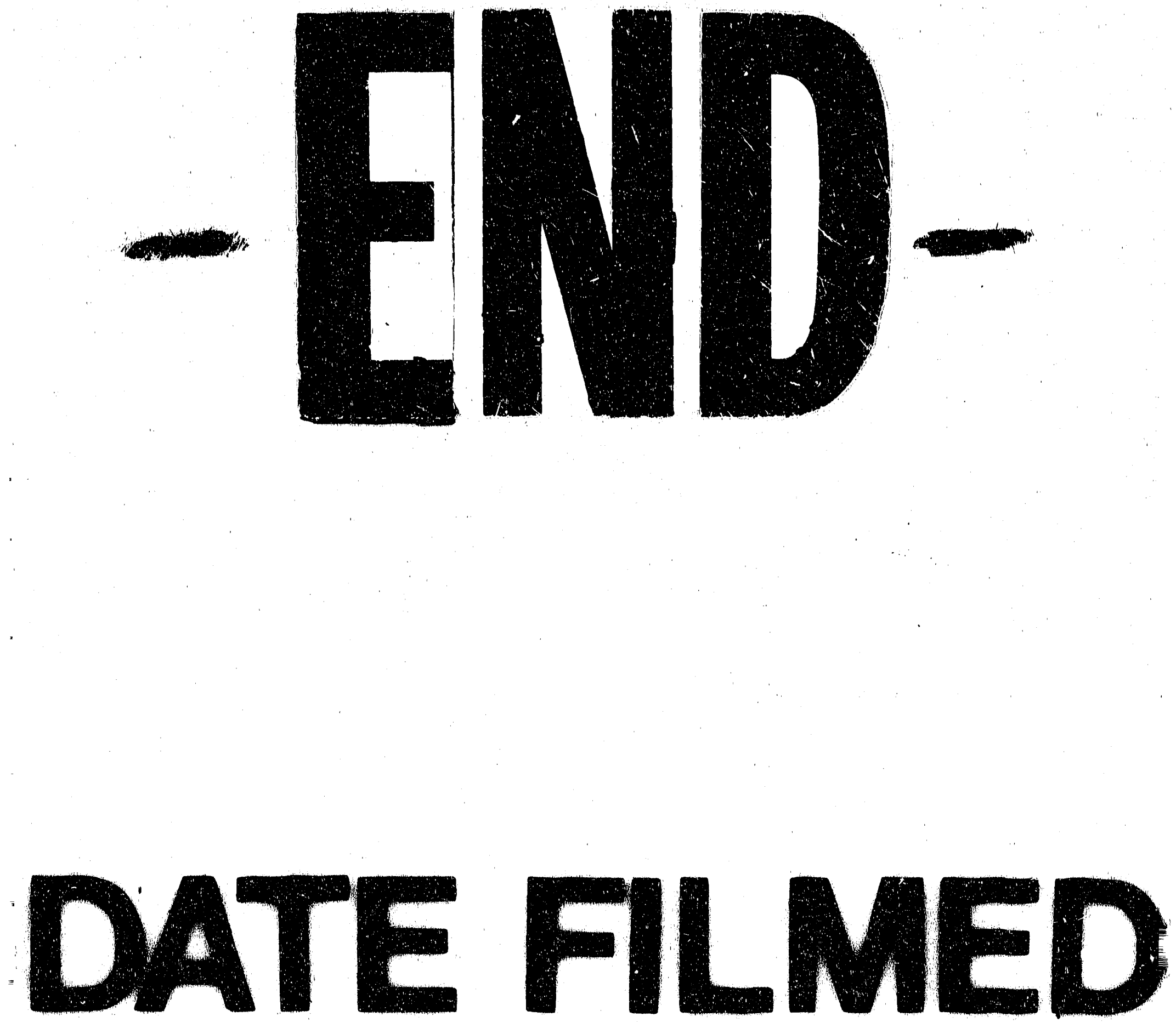

$=$
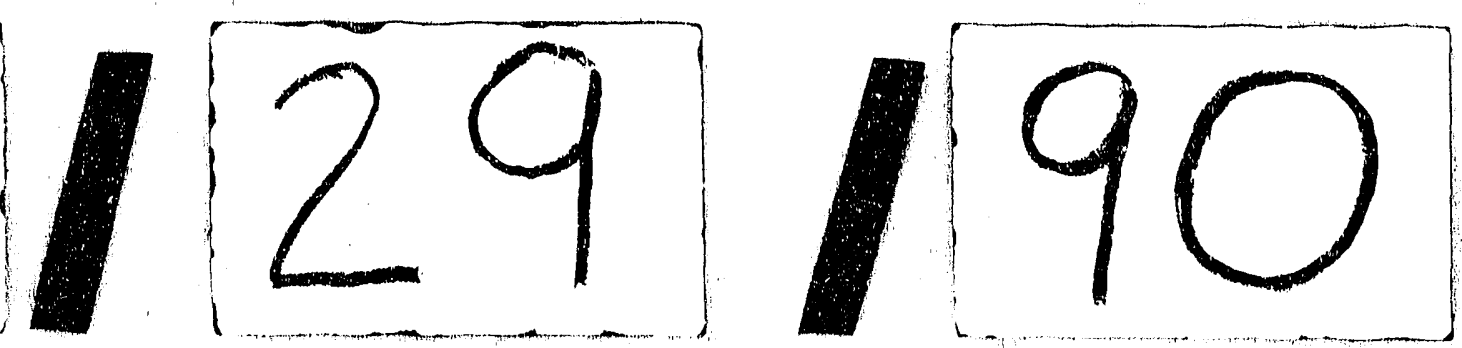